\title{
PIANO NAZIONALE DELLA PREVENZIONE 2005-2007
}

Il 23 Marzo 2005 la Presidenza del Consiglio dei Ministri ha emanato il Piano Nazionale della Prevenzione (PNP) per il Triennio 2005-2007, in attuazione di quanto concordato nella Conferenza Permanente per i rapporti tra lo Stato, le Regioni e le Province Autonome.

Il piano ha quattro finalità generali:

1. la prevenzione cardiovascolare;

2. gli screening oncologici;

3. la prevenzione degli incidenti;

4. il piano delle vaccinazioni.

Alle Regioni ed alle Province è stato fatto obbligo di preparare i relativi Piani Regionale della Prevenzione (PRP) e, entro il 30 giugno 2005, eventuali "progetti pilota" per la valutazione degli effetti (efficacia) della prevenzione.

Le Regioni hanno deciso di effettuare uno sforzo economico considerevole accettando di vincolare all'attuazione del Piano 240 milioni di euro sulla quota del riparto CIPE. Sono state previste ulteriori risorse, per un ammontare di 200 milioni di euro, che le Regioni attingeranno dalla quota indistinta della delibera CIPE per ciascuno degli anni 2005, 2006 e 2007. Le risorse disponibili sono state rapportate al numero degli abitanti di ciascuna Regione.

All'Istituto Superiore di Sanità (ISS) è stato affidato il compito di indicare le linee operative, di verificarne l'applicazione e di valutare i risultati raggiunti tramite il Centro Nazionale per la Prevenzione e il Controllo delle Malattie (CCM), che opera con il supporto di una "task force" di cui fa parte anche Heart Care Foundation.

Per quanto riguarda la prevenzione cardiovascolare il Piano prevede quattro specifici ambiti di interesse:

a) la diffusione della carta del rischio cardiovascolare;

b) la prevenzione dell'obesità nelle donne in età fertile e nel bambino;

c) la prevenzione attiva delle complicanze del diabete di tipo II nell'adulto e nel bambino, aumentando la "compliance" del paziente;

d) la prevenzione delle recidive nei soggetti che hanno già avuto accidenti cardiovascolari, cosicché questi non si ripetano.

Il Dipartimento della Prevenzione e della Comunicazione del Ministero della Salute in una circolare agli Assessorati Regionali alla Sanità, inviata in data 19 ottobre 2005, ha identificato le Linee operative per i PRP e stabilito il 31 Dicembre 2005 come termine ultimo entro il quale le regioni dovranno inviare i progetti relativi al PNP 2005-2007.

Ogni regione deve individuare l'ambito territoriale di attuazione del progetto e le modalità di coor- dinamento. Passare quindi all'adozione della carta del rischio e stabilire il percorso per la gestione del rischio cardiovascolare utilizzando le diverse competenze professionali come la medicina generale e i servizi e le strutture presenti sul territorio che possono svolgere un'azione di controllo sui FR modificabili.

In particolare, per l'attuazione della prevenzione secondaria, il documento ministeriale, facendo riferimento alla letteratura scientifica specifica (EUROASPIRE I e II e GISSI-Prevenzione), così definisce ed articola le linee operative per le pianificazioni:

1. Costituzione, attivazione o rafforzamento della competenza regionale nella prevenzione cardiovascolare (comitato, commissione, struttura regionale ecc.) anche grazie alla collaborazione delle società scientifiche dell'area cardiologica $\mathrm{e}$ della medicina generale.

2. Analisi e definizione del contesto. Ogni regione deve dotarsi di mezzi informativi e decidere come utilizzarli per pianificare e valutare gli eventi. Una possibilità in questo senso è la partecipazione al Registro Eventi Cardiovascolari, coordinato dall'ISS, o realizzare sistemi di registrazione degli eventi coronarici (EC) e degli accidenti cerebrovascolari (AC).

3. Indicazioni alle strutture ospedaliere presenti sul territorio relative alle modalità di dimissioni del paziente infartuato. Ogni paziente dovrebbe essere fornito di un foglio di dimissioni indirizzato al medico curante che specifichi tra l'altro:

- la percentuale del rischio di recidive, valutato attraverso la carta del rischio CV nel postinfarto (GISSI-P);

- indicazioni sulla correzione dei fattori di rischio;

- indicazioni sulla terapia;

- indicazioni sulla riabilitazione;

- indicazione sui controlli periodici da eseguire.

4. Offerta servizi: con la identificazione di strutture che operano per favorire il controllo dei fattori di rischio modificabili (anche nella prevenzione primaria).

5. Piano di formazione del personale sanitario nella prevenzione delle recidive.

6. Programmi educazionali per i pazienti.

7. Individuazione degli indicatori del monitoraggio.

Il PNP, così come impostato, apre ampi spazi all'inserimento del GICR ed è pertanto necessario che il Gruppo si attivi rapidamente ed efficacemente per essere parte integrante del progetto ed avere così il giusto rilievo.

Intanto in alcune regioni, come la Lombardia, il GICR è stato invitato a far parte della costituenda Commissione Regionale per il Piano Prevenzione, insieme ai rappresentanti di altre 30 Società Scientifiche. In altre, come la Campania, sono stati coopta- 
ti dall'Osservatorio Epidemiologico regionale nostri associati come Carlo Vigorito, in rappresentanza del GICR, e Carmine Chieffo, in qualità di Vice-Presidente dell'Heart Care Foundation, istituzionalmente coinvolta nel CCM.

È possibile trovare i piani di azione e d'investimento che ogni regione ha attivato nei siti internet sotto elencati, già inviati da Tommaso Diaco (Referente Regionale della Lombardia) a tutti i componenti del Consiglio Direttivo ed ai Referenti Regionali del GICR. Questi stessi Piani sono stai inviati da Carmine Chieffo anche ai Presidenti Regionali ANMCO ed ai Referenti Regionali di HCF.
Un'azione sinergica e collaborativa delle tre organizzazioni coinvolte certamente potrà produrre risultati di maggior consistenza.

http://pdf.giofil.it/circopdf/cu.zip

www.epicentro.iss.it/focus/piano_prevenzione/ cuore_recidive.asp

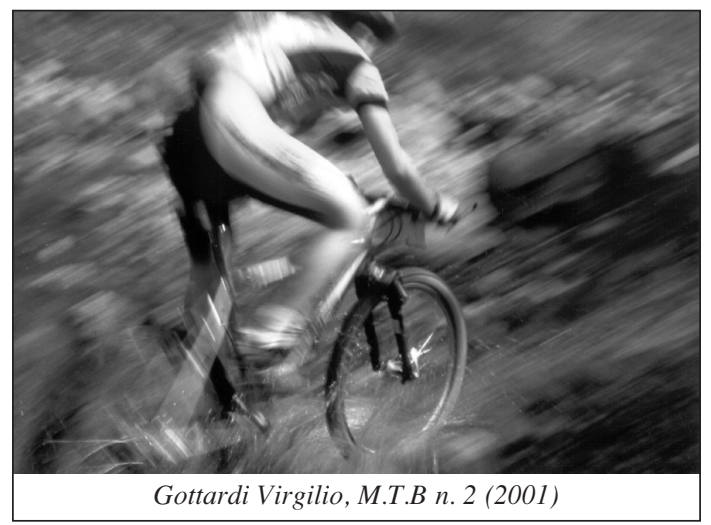

